\title{
O042: Study on adopting the WHO 5-moment of hand hygiene for practices in traditional Chinese medicine (TCM) clinics
}

\author{
P Ching \\ From 2nd International Conference on Prevention and Infection Control (ICPIC 2013) \\ Geneva, Switzerland. 25-28 June 2013
}

\begin{abstract}
Introduction
Hand hygiene $(\mathrm{HH})$ is effective to prevent nosocomial infections in healthcare settings. However, the application of the WHO 5-moment for HH in TCM practices has not been reported. This study was performed to explore the feasibility of applying the WHO 5-moments for $\mathrm{HH}$ using alcohol-based handrub (ABHR) in TCM clinics. The objectives were to study when HH should be practiced, the feasibility of adopting the WHO 5moments and the practicality of using ABHR in TCM practices.
\end{abstract}

\section{Methods}

The clinic was visited to interview TCM practitioners and to understand the different practices performed and the extent of skin and blood and body fluid contacts during TCM practices. Direct observation of practices and videos on each procedure were performed to comprehend $\mathrm{HH}$ opportunities and assess the possibility of adopting the 5-moments. The frequency of $\mathrm{HH}$ action per hour and the need for personal protective equipment were estimated. Possible placement of AHR was identified.

\section{Results}

The patient care and treatment practices in the TCM are different from western medicine and include: visit to a TCM practitioner, acupuncture with electric stimulation, moxibustion, cupping, and massage. TCM practitioner consultation, cupping and massage only involve skin contact and moment 1 and 4 are required. Acupuncture and moxibustion may cause limited blood exposure and moments 1, 3, 4 and 5 of $\mathrm{HH}$ are required after glove removal. Acupuncture, moxibustion and cupping require $\mathrm{HH}$ when applying related devices and upon removal. Practitioner consultations might need 3-4 $\mathrm{HH}$ actions per hour. Other treatments duration last 30 to $60 \mathrm{~min}$ utes and thus $\mathrm{HH}$ frequency is 1-2 times per hour. ABHR can be conveniently placed at couch and treatment trolley for use.

\section{Conclusion}

The study demonstrates that TCM include 5 treatment practices that are different from western medicine. Limited blood exposures would occur during acupuncture and moxibustion while moments $1,3,4 \& 5$ are frequently recorded. The $\mathrm{HH}$ opportunities range from 2 to 4 per hour and are practicable. This preliminary study anticipated that $\mathrm{HH}$ using ABHR is doable in TCM clinic and WHO 5-moments for HH can be applied in the TCM practices. Tool kit and education for TCM practitioners will be developed. Further studies and pilot implementation will be conducted.

\section{Disclosure of interest}

None declared.

Published: 20 June 2013

doi:10.1186/2047-2994-2-S1-042

Cite this article as: Ching: O042: Study on adopting the WHO 5moment of hand hygiene for practices in traditional Chinese medicine (TCM) clinics. Antimicrobial Resistance and Infection Control 2013 2(Suppl 1): 042.

Hong Kong Baptist Hospital, Hong Kong, Hong Kong

@ 2013 Ching; licensee BioMed Central Ltd. This is an Open Access article distributed under the terms of the Creative Commons Attribution License (http://creativecommons.org/licenses/by/2.0), which permits unrestricted use, distribution, and reproduction in any medium, provided the original work is properly cited. 